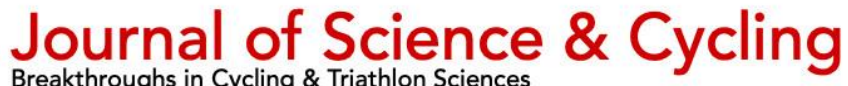

Editorial

\section{Power Profiling in Professional Road Cycling - the past, the present and the future}

\author{
Peter Leo ${ }^{1}$ James Spragg ${ }^{2}$ and Iñigo Mujika ${ }^{3,4}$ \\ 1 Department Sport Science University of Innsbruck, Innsbruck, Austria \\ 2 HPALS, Department of Human Biology, University of Cape Town \\ 3 Department of Physiology, Faculty of Medicine and Nursing, University of the Basque Country, Leioa, Basque Country \\ 4 Exercise Science Laboratory, School of Kinesiology, Faculty of Medicine, Universidad Finis Terrae, Santiago, Chile \\ * Correspondence: Peter Leo; peter.leo@uibk.ac.at \\ Published: 30 June 2021
}

Keywords: power output, racing, performance, modelling

\section{The past}

Power profiling has always been an area that has received great interest within cycling specific research (Leo et al., 2021; Pinot \& Grappe, 2011; Quod et al., 2010; Sanders \& van Erp, 2021). Although there are numerous methodologies that fall under the umbrella of power profiling, there remains limited consensus on testing protocols, modelling procedures and practical applications. Arguably the most used methodology is deriving mean maximum power outputs MMP values from training and racing. This may be, at least partially, due to the ease in which MMP values can be derived. However, this approach does have several limitations. MMP data can include submaximal data, unstandardized effort lengths and changing environmental conditions (e.g., sea level vs. altitude). Even though MMP data have shown to be a good predictor of race typology, difficulty and performance (Leo, et al. 2021a; 2021b; Sanders and van Erp 2021), little attention is often paid to the accuracy and reliability of the power output measurement. Since the first crank spindle manufactured power meters from SRM in the 1980s many other companies came up with a similar product measuring power output via crank arms, spindles, and pedals. Despite the power meters' popularity, little information is known about the accurate calibration process to ensure power meters measure valid and reliable. However, this is of paramount importance for all further power output data processing and analysis. Manual calibration of a power meter according to Wooles et al. (2005) is limited by most manufacturers due to their firmware policies and intellectual property rules. Instead, power meter manufacturers provide their internal "arbitrary" calibration process, which reveal little information for customers. In the authors' opinion it would be advantageous that power meters allow a manual adjustment of the slope and zero-offset alongside the automatic calibration process, (e.g., via a mobile smartphone app), to ensure accurate and reliable power output readings.

\section{The present}

An interesting current avenue of research in the field of power profiling is to look at the decline in MMP values after accumulated work. This approach has been used in recent research (van Erp, Sanders, and Lamberts 2021; Leo, Spragg, Simon, Mujika, et al. 2021; Muriel et al. 2021) which has given some great insights into the role fatigue resistance plays in cycling performance. It has even been suggested adding the "fatigue resistance" component as the fourth component of Joyner and Coyle's endurance performance model (Joyner and Coyle 2008) 
alongside maximum oxygen uptake, the submaximal threshold boundaries (e.g., gas exchange threshold and critical power) and working economy/efficiency. This highlights the impact that power profiling can have on our understanding of the performance determinants of cycling. One could argue that all we have achieved is a better quantification of what coaches have known for years, be that what it may all hypotheses need to be rigorously tested!

\section{The future}

Looking to the future there are many avenues into which the field of power profiling can tread. Power profiling has historically helped researcher to first describe, and more recently to (better) explain performance in cycling. However, thus far, one major limitation of power profiling has been its detachment from what is happening in the heart of the peloton. Researchers are often limited in their ability to draw conclusions because of the potential influence of how race tactics are being deployed. However, rather than seeing this as a limitation it can be viewed as an opportunity. Performance is always multifacetted. It arises from the interplay of numerous factors including power production, tactics, psychology, social structures, biomechanics and many more. One could argue that the real efficacy of power profiling will come when the measured power output values are considered in conjunction with other factors. A great example of this is a recent paper by van Erp, Kittel, and Lamberts 2021 whereby the influence of different race tactics on power output prior to a sprint finish were analysed in partnership with the athlete that had produced them. This more nuanced approach is arguably a more valuable one. Not only because it gives a richer context to power meter data, but it can also help to translate the findings of any research back into the world of professional cycling.

The future is bright so long as the field of power profiling continues to not only further our understanding of performance, but also shapes, and improves performance.
Therefore, we encourage all efforts to achieve these objectives.

\section{Conflict of Interest}

The authors declare that this editorial was written following invitation from the chief editor and is in the absence of any commercial or financial relationship that could be construed as a potential conflict of interest.

\section{Author Contributions}

P.L. J.S. and I.M. equally contributed to the writing of the editorial and all authors agreed to its final version.

\section{Funding}

No funding was provided for this article.

\section{Acknowledgments}

The authors would like to thank the chief editor for the opportunity to write this editorial.

\section{References}

Leo, P., Spragg, J., Menz, V., Mujika, I., \& Lawley, S. J. (2021). Power profiling in U23 professional cyclists during a competitive season. Int J Sports Physiol Perform, 16(6), 881-889. https://doi.org/10.1123/ijspp.2020-0200

Leo, P., Spragg, J., Podlogar, T., Lawley, J. S., \& Mujika, I. (2021). Power profiling and the power-duration relationship in cycling: a narrative review. Eur J Appl Physiol, aop, 1-16.

Leo, P., Spragg, J., Simon, D., Lawley, S. J., \& Mujika, I. (2020). Training Characteristics and Power Profile of Professional U23 Cyclists throughout a Competitive Season. Sports, 8(12), 167. https://doi.org/https://doi.org/10.3390/s ports 8120167 
Leo, P., Spragg, J., Simon, D., Mujika, I., \& Lawley, S. J. (2021). Power profiling, workload characteristics and race performance of U23 and professional cyclists during the multistage race Tour of the Alps. Int J Sports Physiol Perform, in press. https://doi.org/https://doi.org/10.1123/ij spp.2020-0381

Pinot, J., \& Grappe, F. (2011). The record power profile to assess performance in elite cyclists. Int J Sports Med, 32(11), 839-844. https://doi.org/10.1055/s-00311279773

Quod, M. J., Martin, D. T., Martin, J. C., \& Laursen, P. B. (2010). The power profile predicts road cycling MMP. Int J Sports Med, 31(6), 397-401. https://doi.org/10.1055/s-0030-1247528

Sanders, D., \& van Erp, T. (2021). The physical demands and power profile of professional Men's cycling races: An updated review. Int J Sports Physiol Perform, 16(1), 3-12. https://doi.org/10.1123/IJSPP.2020-0508 